\title{
DETERMINATON OF THE SPATIAL ENERGY DISTRIBUTION GENERATED BY MEANS OF A FLASH LAMP
}

\author{
R. Krankenhagen and Ch. Maierhofer
}

BAM Federal Institute for Materials Research and Testing, Berlin, Germany, Rainer.Krankenhagen@bam.de Mail: Unter den Eichen 87, 12205 Berlin, Germany; Phone: +49 308104 3784; Fax: +49 3081041847

\begin{abstract}
In order to carry out a quantitative analysis of thermographic data the heating energy and its spatial distribution have to be known. Especially in the case of flash lamp excitation the correct determination of these parameters is complicated due to very short excitation times combined with high power densities. The paper describes a procedure for the characterization of such lamps using a simple calorimetric sensor and a plane reference area. The presented results illustrate the performance and the suitability of the method in the field of thermography.
\end{abstract}

\section{Introduction}

Active thermography plays an increasing role within the various methods of non-destructive testing (NDT). This development is driven by the permanent further development of the different digital technologies of infrared cameras. However, the implementation of a testing procedure requires not only a suited infrared camera, but also qualified heat sources to introduce the needed amount of energy into the probe under test. Very common sources are flash lamps [1], because they enable very fast heating impulses in order to characterize "thermal shallow" defects. Drawbacks of such lamps are the limited energy per shot, durability and health and safety requirements.

Due to the functional principle of discharge lamps the light emitting surface is limited to small areas. Therefore, the light source itself is more or less point-shaped. The resulting energy distribution of such a point-shaped source on a plane surface is non-uniform which is unfavourable for the detection of flares below the surface [2]. A common way for reducing the influence of an inhomogeneous excitation is the application of time-based evaluation methods like pulsephase-thermography or lock-in techniques. However, it is advantageous to reduce spatial inhomogeneities within the excitation process as much as possible. This can be achieved by the application of beam shaping elements. In other cases, the evaluation of the measurement results is complemented with simulations of the process, where the nonuniform thermal excitation is needed to be known as input parameter explicitly [3].

The aim of the paper is the description of a procedure for exploring energy distributions on a plane surface originated by flash lamps. This procedure is based on a very simple calorimetric sensor. It will be shown, that this sensor works quite well as an absolute energy meter. The combination of this sensor with a black sheet of paperboard enables the investigator to study energy distributions in detail. This concept has some similarities with the method applied by S. Pickering and D. Almond [4], where the temperature decay at an undistorted part of the sample itself was measured. If the sample is "thermally thick" and the effusivity of the sample material is known, the input energy can be estimated. However, from a methodical point of view it is unfavourable to use the sample to be probed as a reference for the measurement.

\section{Sensor concept}

The used simple concept to determine spatial energy distribution includes a large size flat test area for relative measurements and a small size energy sensor to obtain an absolute value [2]. This absolute value allows the shift of the relative distribution along the energy axis to the correct scale. Both components will be read out simultaneously using an infrared camera, which is already present for thermography investigations.

The realization of a large size energy monitor was carried out in such a way, that a black paperboard (98 $\mathrm{cm} \times 70 \mathrm{~cm}$ area, $0.4 \mathrm{~mm}$ thick) was clamped at the top edge. So, the whole paperboard hung down freely. In order to measure the energy of a flash persisting only few milliseconds, a fast sensor with wide-band absorption coating and energy calibration has to be used [3]. But, correctly calibrated energy values can be obtained merely in the case of parallel light beams with an aperture smaller than the sensor area. Otherwise, the window at the sensor housing acts as an aperture influencing the angular distribution of the incoming light. Therefore, in order to obtain a proper energy value inside an arbitrary light radiation field a sensor is required with no aperture at all.

Therefore, an aperture-less sensor was realized as a calorimeter: a small metal plate of precisely defined shape with known thermal properties and black coating should be exhibit a certain temperature rise after UV-, VIS- or IR- 
radiation. This temperature rise can be observed by means of the infrared camera easily. The main properties of the investigated metal plates are summarized in the following table 1:

Table 1: properties of the designed metal plates used as calorimetric sensors

\begin{tabular}{|l|l|l|}
\hline \multicolumn{1}{|c|}{ Name } & HS 1 & HS 2 \\
\hline Material & V2A steel & V2A steel \\
\hline sensor area & $1 \mathrm{~cm}^{2}$ & $1 \mathrm{~cm}^{2}$ \\
\hline Thickness & $1 \mathrm{~mm}$ & $2 \mathrm{~mm}$ \\
\hline Mass & $0.774 \mathrm{~g}$ & $1.54 \mathrm{~g}$ \\
\hline thermal capacity & $0.369 \mathrm{Ws} / \mathrm{K}$ & $0.730 \mathrm{Ws} / \mathrm{K}$ \\
\hline
\end{tabular}

Figure 1 shows the front side of both sensors each with an area of $1 \mathrm{~cm}^{2}$. A small wooden stick is anchored at the back side to provide the sensor mounting in an experimental setup. This kind of fixing should minimize conductive energy losses. Figure 2 offers a lateral view on the sensors attached to a block of white rigid foam.

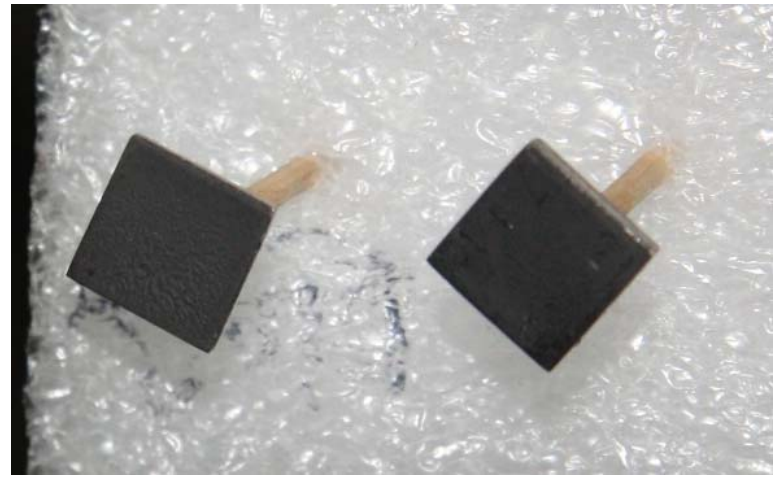

Fig. 1: front view on the investigated calorimetric sensors, wood sticks at the back side permit the fixation, fixation was conducted on a block of white rigid foam

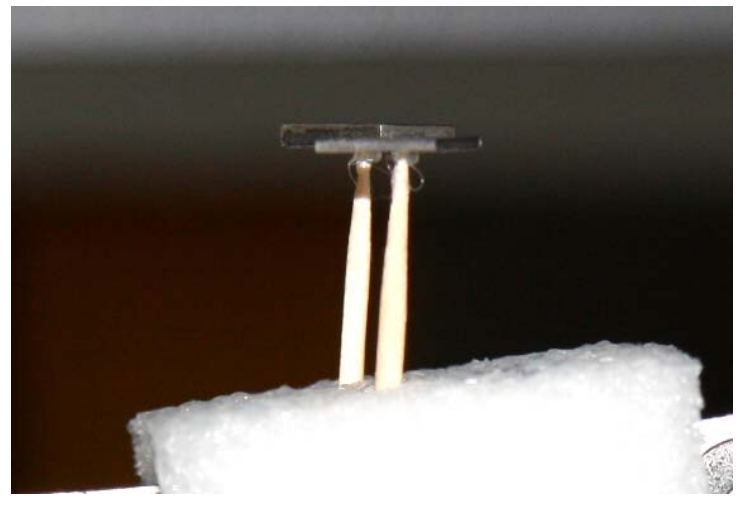

Fig. 2: lateral view on the investigated sensor configuration

Though the sensor concept is simple from a theoretical point of view, the authors wanted to verify the suitability of these plates for monitoring energy inputs experimentally. This could be achieved by a systematic investigation of the sensor signal, i.e. the temperature rise, caused by a well-defined energy source. The following task list comprises the issues to be considered:
A) Stable and detectable value of the temperature rise
B) Proportionality between temperature rise and energy input
C) Agreement between the theoretical and the measured value of the thermal capacity of the sensor (requires well defined energy inputs)
D) Assignment of relative spatial temperature distributions with the absolute energy density at one point inside the excited zone

\section{Experimental setup}

As a source for well defined energy inputs a calibrated blackbody radiator with a diameter of $15 \mathrm{~cm}$ was selected (Fluke model 4180). Energy variations can be reached in two different ways: by changing the radiation time or by changing the surface temperature resp. the power of the radiator. However, placing a power sensor directly in front of such a blackbody radiator one will not measure the power density described by the Stefan-Boltzmann-law. This is due to the fact, that the plane radiating area is limited and cannot comprise the whole half-space. But, under certain conditions a simple formula can be used to calculate the incident radiation: 


$$
M=M_{0} \cdot \sin ^{2}(\alpha) \quad[M]=\mathrm{mW} / \mathrm{cm}^{2}
$$

where $M$ is the power density at the sensor position, $M_{0}$ is the power density given by the Stefan-Boltzmann-law and $\alpha$ is the half of the aperture angle which is formed by the circular radiating zone with respect to the sensor [5]. The following conditions must be fulfilled in order to apply Eq.(1) correctly:

i) the radiating area is circular

ii) the detector is located along the optical axis

iii) the area of the detector is very small in relation to the radiating area

iv) the detector must not have any kind of aperture

The geometrical relations are shown in figure 3 schematically. It is obvious that our calorimetric sensors meet requirements iii) and iv), whereas the used radiator agrees with i).

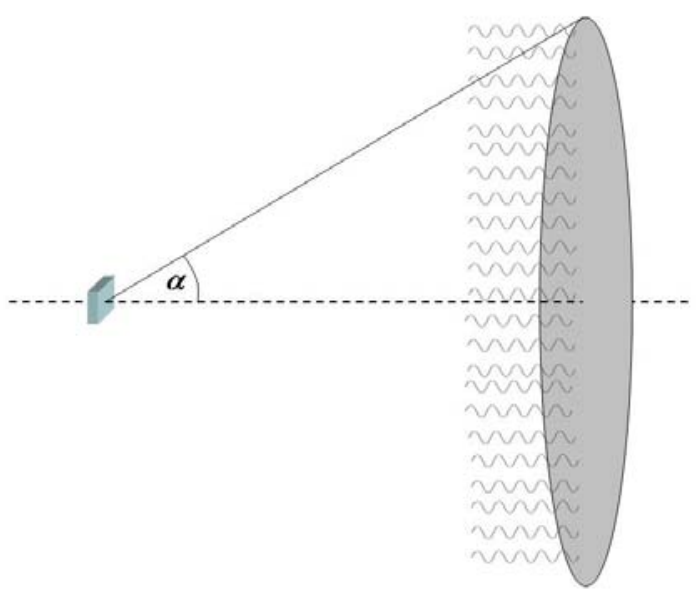

Fig. 3: setup with typical geometrical relations necessary for using Eq.(1) correctly

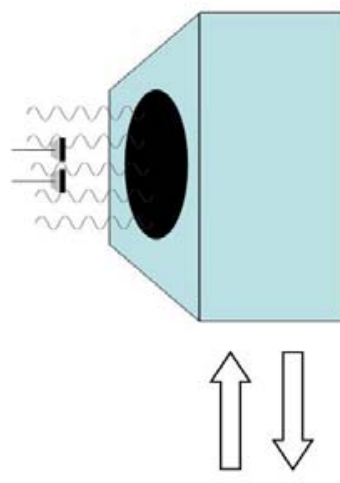

Fig. 4: scheme of the setup, the black body radiator was moved in front of the sensors (irradiating position), after the heating time it was removed enabling the observation of the sensors by the infrared camera (observation position)

A crucial drawback of the blackbody radiator is the limited temperature range (max. $120^{\circ} \mathrm{C}$ ) leading to a restricted energy output. Considering the background radiation at $20^{\circ} \mathrm{C}$ a maximum power density of $94 \mathrm{~mW} / \mathrm{cm}^{2} \mathrm{can}$ be earned. In order to obtain an energy control range as large as possible the sensor should be located nearby the radiating area. But, in such a configuration the front side of the calorimetric sensor is covered by the radiator itself and cannot be investigated by the infrared camera. Figure 4 illustrates this situation. We performed the experiments in such a way, that the blackbody radiator was moved quickly in between the sensors and the camera. After staying in this "radiating position" for the specified time the radiation source was removed to the "observation position" opening the view onto the sensor surfaces. In figure 4 , these movements are indicated by the two arrows. Figure 5 gives an impression of the entire experimental setup realized in our laboratory.

It should be noted, that the infrared camera must be situated in such a distance that the sensor plate area will cover a sufficient number of pixels in order to minimize the influence of lateral crosstalk effects inside the pixel array. During our measurements the distance between the calorimetric sensors and the camera lens (FOV: $30^{\circ} \times 23^{\circ}$ ) was approximately $45 \mathrm{~cm}$ leading to a square of 30 pixels side length within the infrared image. 


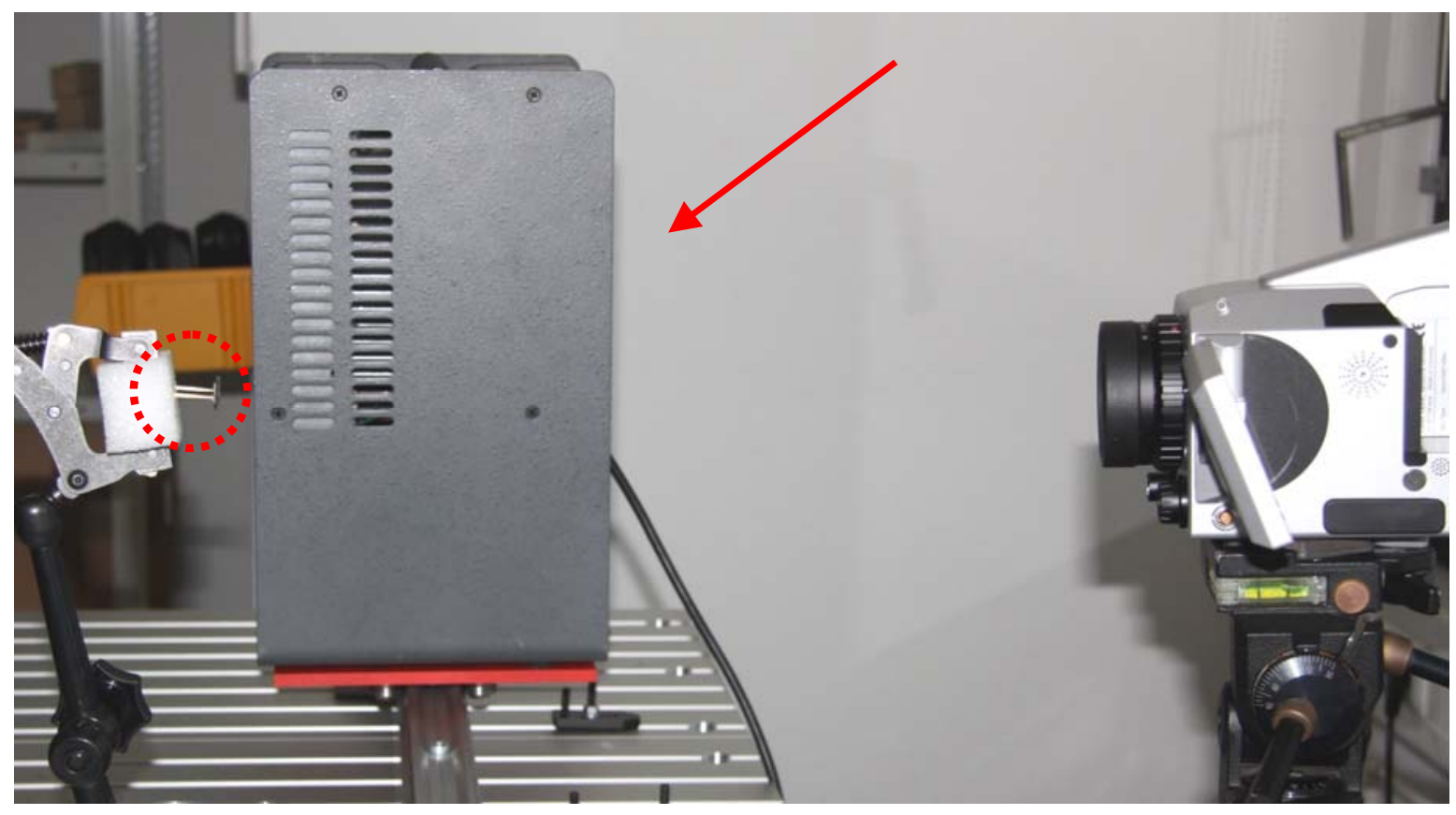

Fig. 5: experimental setup, the red circle surrounds both calorimetric sensors, the arrow points to the blackbody radiator mounted on a carriage plate, the infrared camera is situated on the right hand side

According to the main concept the temperature rise at the calorimetric sensor should be detected by means of an infrared camera. Our investigations were performed with the uncooled bolometer camera VarioCam hr. The most important technical specifications are:

- $640 \times 480$ pixel array

- $50 \mathrm{~Hz}$ frame rate

- $\quad 0.08 \mathrm{~K} \mathrm{NETD}$

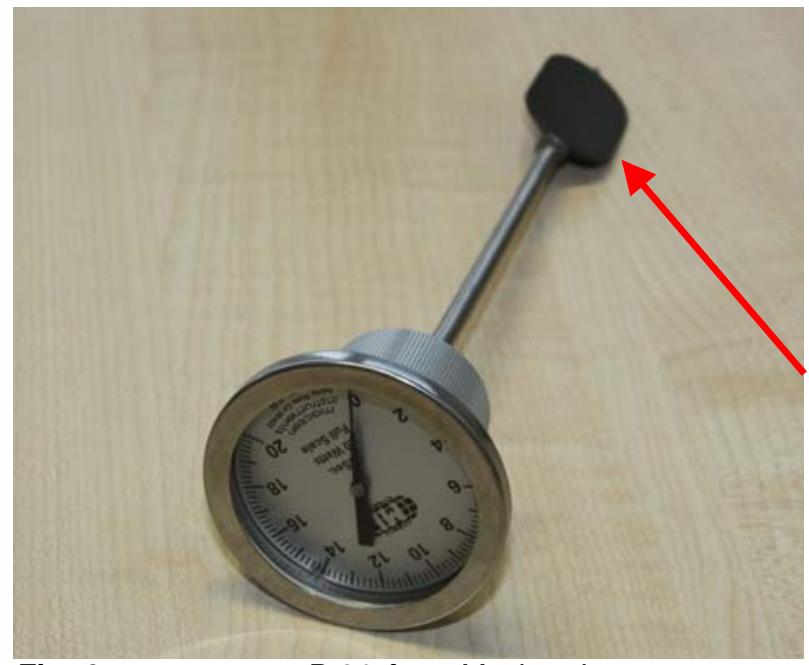

Fig. 6: power meter $P 20$ from Macken Instruments, the arrow points to the sensitive zone

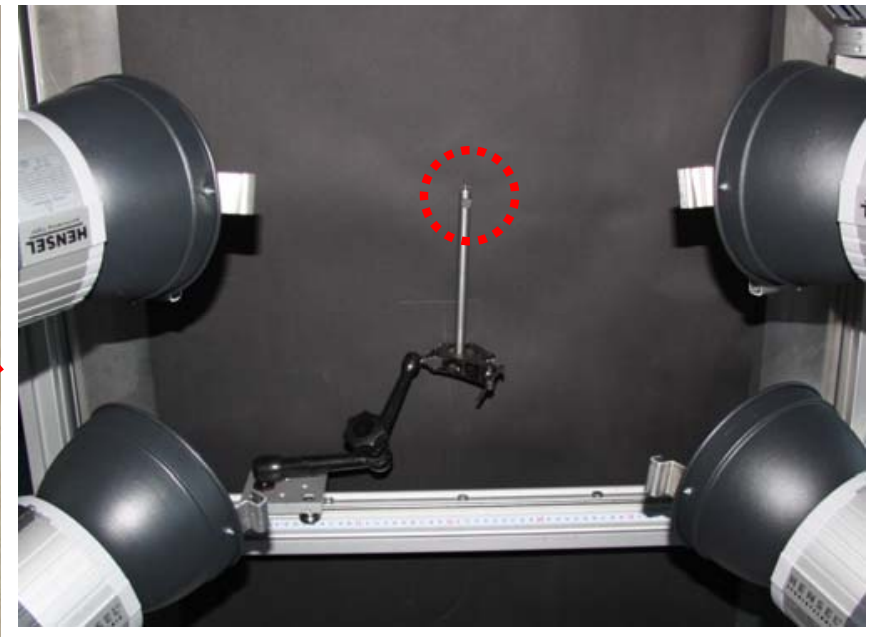

Fig. 7: array of 4 flash lamps for thermal flash excitation of larger areas, the red circle includes the calorimetric sensor located directly in front of the black paperboard in the back

In order to verify the true power density and the validity of Eq.1 a pure mechanical power meter P 20 from Macken Instruments was used (see figure 6). The main advantage of this device is the lack of any kind of housing around the 
sensitive zone. So, no aperture effects have to be regarded and Eq.1 should be valid if the sensitive zone is vertically oriented and directly placed on the optical axis.

Flash light excitation was carried out by means of one or more EH Pro 6000 heads of HENSEL Studiotechnik with back side reflector, which has an aperture diameter of $17 \mathrm{~cm}$. The maximum input energy is $6 \mathrm{~kJ}$ per shot and can be reduced continuously down to $3 \%$. Figure 7 shows an array of 4 of these flash lamps in our laboratory.

\section{Experimental results}

\subsection{Thermal excitation with blackbody radiator}

Generally, the temperature rise caused by an external energy input can only be evaluated, if the temperature level remains constant after the heating period (point $A$ of the task list). However, any heated sample that is not in thermal equilibrium with its environment will cool down immediately after removing the external heat source. So, it is an essential point how stable the reached temperature at the plates will be. Figure 8 shows a thermogram of the rigid foam block with both sensors on it, shortly after the irradiation and after subtracting the thermogram recorded before heating. As to be expected, the thinner plate with smaller thermal capacity (HS1) appears warmer in comparison to the other one (HS2). Please note that the surface temperature of the foam block in the back reached a considerable temperature increase of 4 $\mathrm{K}$, which is outside the displayed temperature range in figure 8 . This was mainly due to the sufficient smaller heat capacity of the foam. In figure 9 , one can see the related temperature development within a time period of $5 \mathrm{~s}$. The zero point at the time axis corresponds to the first image after removing the radiator. The temperature development can be divided into two regions: a fast decay within the first $300 \mathrm{~ms}$ followed by a very slow decrease superimposed by smaller drift effects of the infrared camera. That means, after $0.3 \mathrm{~s}$ the surface temperature of the calorimetric sensors, measured by the infrared camera, remains approximately constant. Furthermore, the value of the measured temperature difference lies in the expected range: $50 \mathrm{~mW} / \mathrm{cm}^{2}$ for $10 \mathrm{~s}$ generates an energy input of $0.5 \mathrm{Ws} / \mathrm{cm}^{2}$, which should induce an increase of 1.3 $\mathrm{K}$ in the case of HS 1 and $0.65 \mathrm{~K}$ for HS2 (assuming thermal capacities shown in table 1). These values are close to the experimental data shown in figure $9(1.4 \mathrm{~K}$ for HS1 and $0.85 \mathrm{~K}$ for HS2). These preliminary results are the basis for further investigations.

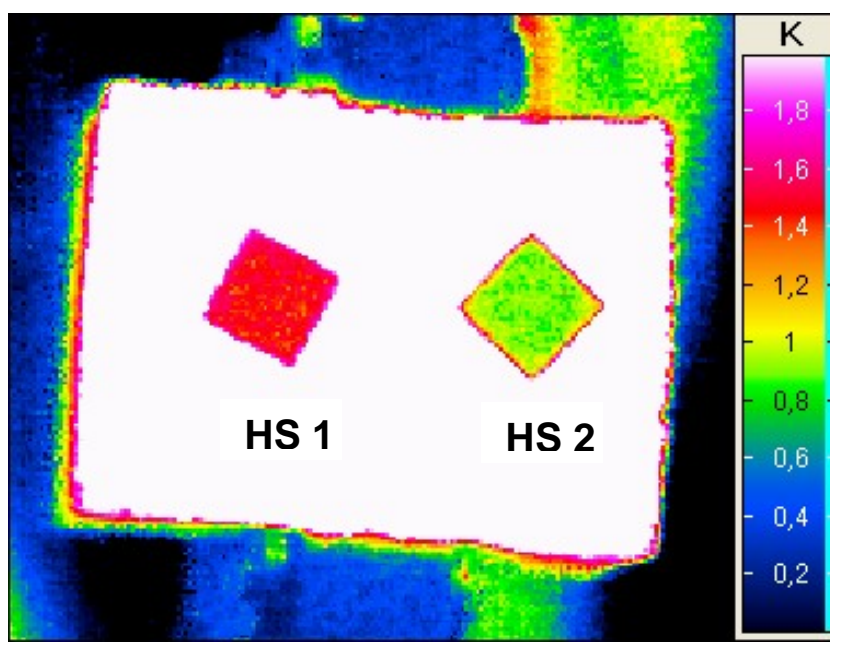

Fig. 8: difference thermogram of both calorimetric sensors after $10 \mathrm{~s}$ radiation induced by a blackbody radiator at $120^{\circ} \mathrm{C}$ in $4.5 \mathrm{~cm}$ distance

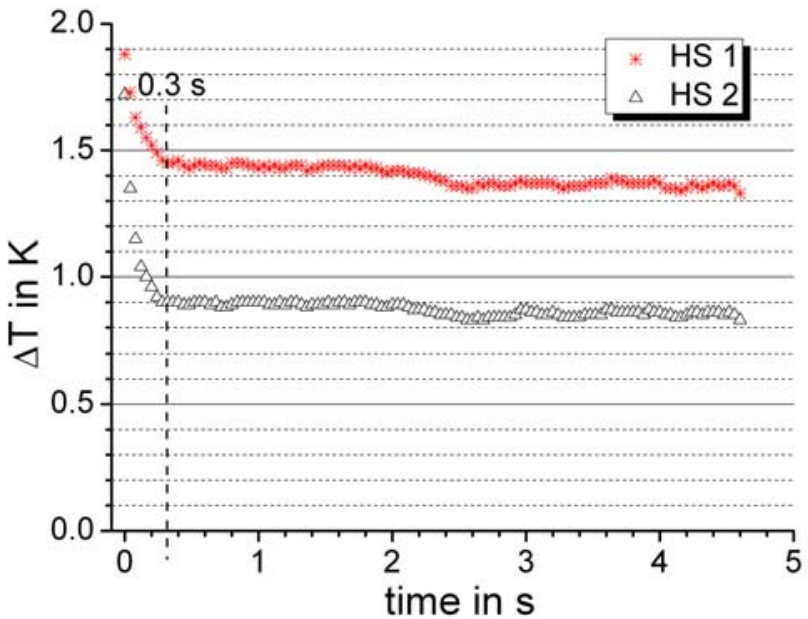

Fig. 9: temperature decay at the surfaces of the calorimetric sensors after $10 \mathrm{~s}$ radiation induced by a blackbody radiator at $120^{\circ} \mathrm{C}$ in $4.5 \mathrm{~cm}$ distance

Point B of our task list requires a direct proportionality between the energy input and the resulting temperature rise. Like mentioned before, the energy input caused by a blackbody radiator can be varied in two different ways: changing the power during a certain radiation time and vice versa. Also in [5] a blackbody radiator was used for the characterization of commercial radiometers. Figure 10 includes the experimental results for variable power densities. 


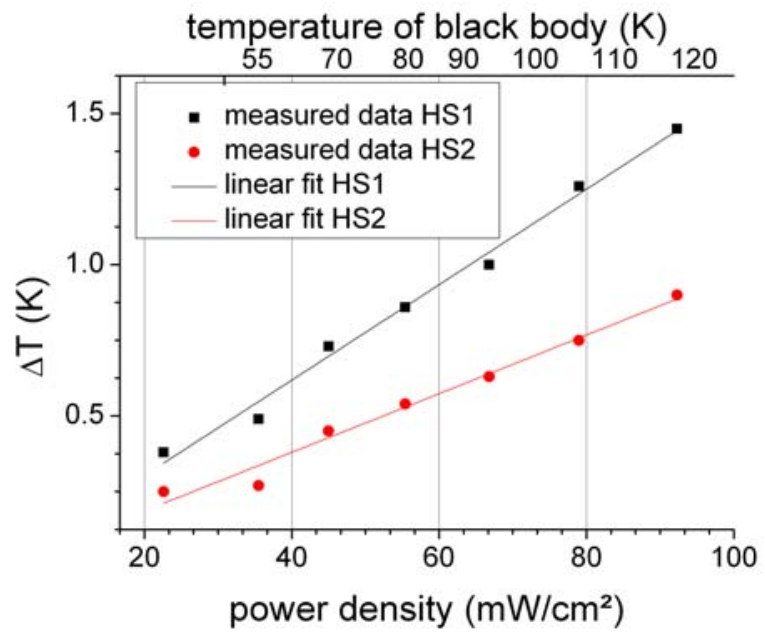

Fig. 10: temperature increase at different input energies on both calorimetric sensors, measured $0.3 \mathrm{~s}$ after the thermal excitation; energy variation was realized by changing the surface temperature of the blackbody radiator in $4.5 \mathrm{~cm}$ distance while the radiation time has been kept constant at $10 \mathrm{~s}$

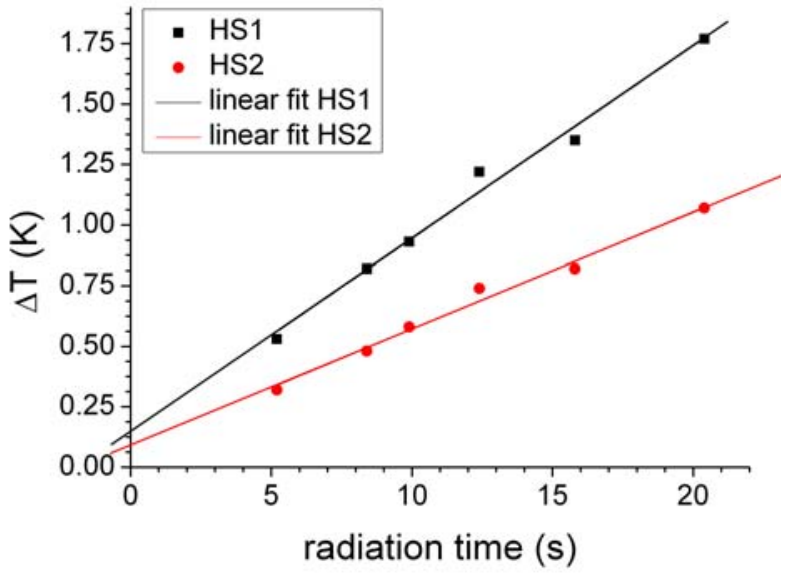

Fig. 11: temperature increase at different input energies on both calorimetric sensors measured $0.3 \mathrm{~s}$ after the thermal excitation, energy variation was realized by changing the radiation time, while the temperature of the blackbody radiator in $4.5 \mathrm{~cm}$ distance has been kept constant at $100^{\circ} \mathrm{C}$

The linearity is obvious for both sensors. It should be noted that the values, plotted on the x-axis, are not the energy densities in the plane of the sensor surface but directly at the radiator surface. Therefore, these values represent a theoretical upper limit for the true energy at any position outside. Figure 11 shows the results of a corresponding measurement series, where the radiation time was varied and the blackbody temperature remains constant. Again, a linear correlation could be found. Interestingly, the linear fits exhibit a positive offset at $t=0$ which seems to be unreasonable. This effect is probably due to the movements of the radiator in and out of the optical axis, which lead to an undefined additional energy input.

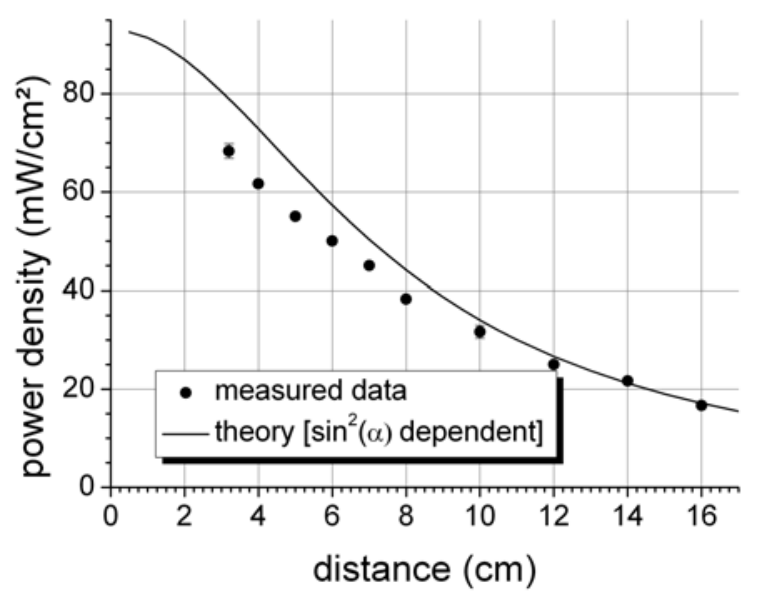

Fig. 12: power densities of the blackbody radiator at $120^{\circ} \mathrm{C}$, measured in different distances along the optical axis using sensor $P$ 20, the solid line indicates the theoretical curve

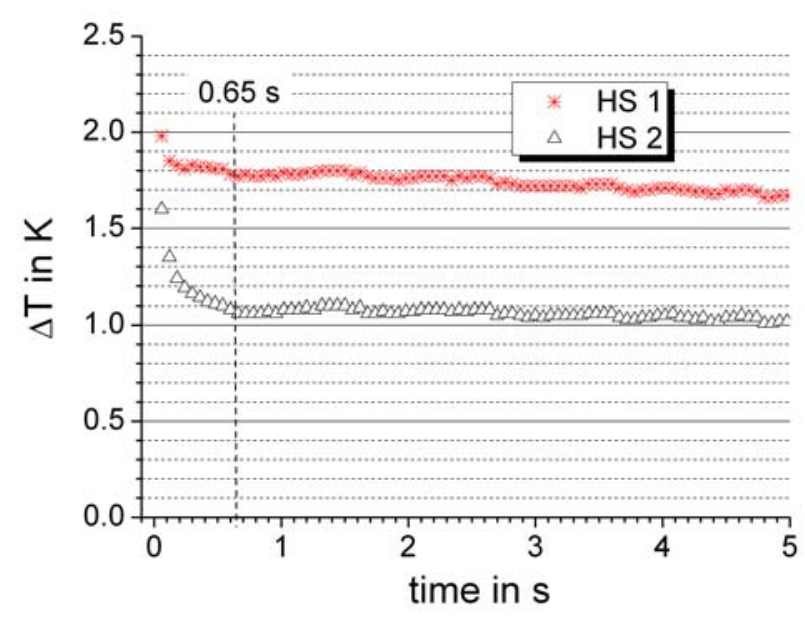

Fig. 13: temperature decay at the surfaces of the calorimetric sensors after $6 \mathrm{~kJ}$ flash excitation in $37 \mathrm{~cm}$ distance

According to the suggested sensor concept these calorimetric sensors should measure the true energy input. For verification, a comparison between the actually incident energy and the determined energy has to be conducted (point $\mathrm{C}$ 
of the task list). In order to check the validity of Eq.1 for determining the radiated energy of the blackbody radiator the $\mathrm{P} 20$ was vertically oriented and directly placed on the optical axis. The power was measured in different distances to the radiator. The results of this investigation are depicted in figure 12. There is a good agreement between theory (Eq. 1) and experiment at longer distances down to $10 \mathrm{~cm}$ (further discussion see below).

\subsection{Thermal excitation with flash lamps}

After the evaluation of the calorimetric sensors by means of the blackbody radiator the sensor performance had to be checked in the case of flash excitation. Concerning this point there are two questions:

i) Is the thermal capacity of the sensors sufficient small to measure the energy input in typical flash lamp setups (configurations)?

ii) Is the temperature increase stable enough to determine an appropriate value?

Both questions could be answered positively, as one can see in figure 13. Again, the curves can be divided into the two time periods. The slow part can be used for the estimation of the temperature rise. However, in comparison to figure 9, it needs more waiting time until the surface temperature is stabilized.

The last step for establishing the suggested sensor concept is placing a calorimetric sensor immediately in front of the black paperboard and observing the whole scene by an infrared camera. Figure 14 demonstrates the result of such an experiment, where the geometrical energy distribution of a single flash lamp was investigated. The flash lamp aperture was covered by a Plexiglas plate to prevent an additional long term energy output due to the "after glow" of the lamp tube. Corresponding to the temperature distribution the observed energy is distributed with circular symmetry. Combining the relative temperature difference with the absolute energy value of the calorimetric sensor a radial energy distribution can be evaluated (shown in figure 15). The level of energy input is similar to this of S. Pickering et.al., who reported an energy density $1.2 \mathrm{~J} / \mathrm{cm}^{2}$ generated by $12 \mathrm{~kJ}$ flash lamp system [4].

In order to enhance the energy input and increase the excited area the application of flash lamp arrays is possible. Figure 16 offers an impression of the energy distribution of a four lamp setup (shown in figure 7). It can be seen that the spatial distribution is not homogeneous, a local minimum was formed inside the excited zone. Directly to the right the corresponding energy profile along the yellow line is depicted (figure 17). The profile verifies that the power density within the excited zone fluctuates by less then $20 \%$. Thus, there seems to be some potential for improving the homogeneity by optimizing the lamp positions and orientations.

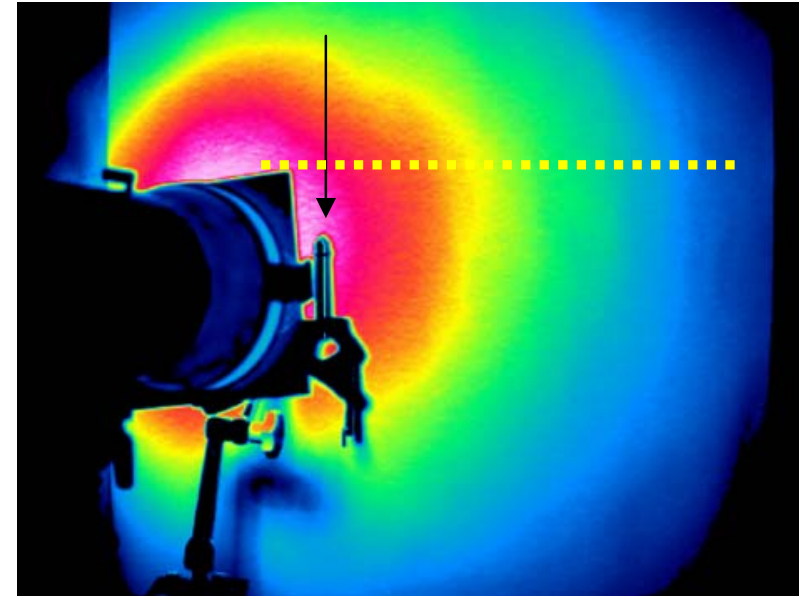

Fig. 14: spatial energy distribution $20 \mathrm{~ms}$ after the $6 \mathrm{~kJ}$ flash of a flash lamp in $35 \mathrm{~cm}$ distance and recorded by an infrared camera, the arrow points to the calorimetric sensor

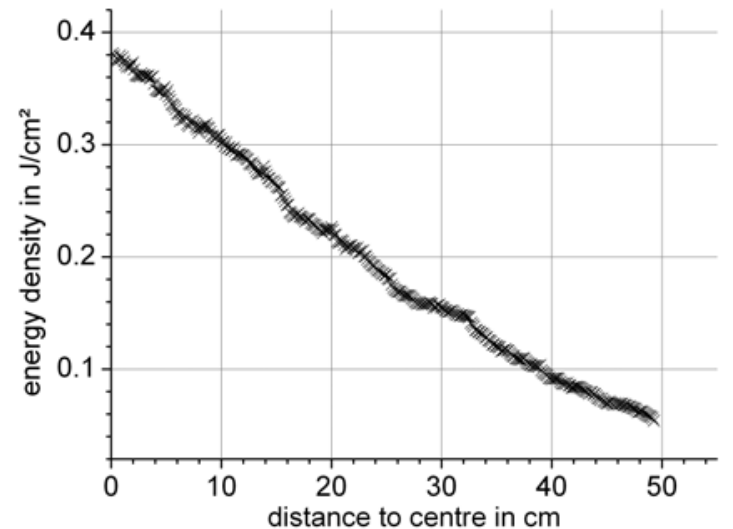

Fig. 15: profile of the energy density of a flash lamp, determined along the yellow dotted line in figure 14 


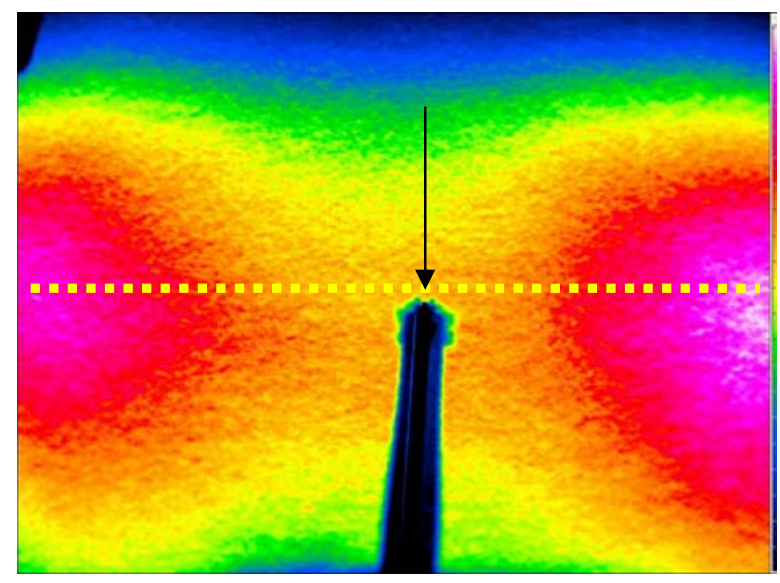

Fig. 16: spatial energy distribution $20 \mathrm{~ms}$ after the flash of a four lamp array in $40 \mathrm{~cm}$ distance and recorded by an infrared camera, the arrow points to the calorimetric sensor

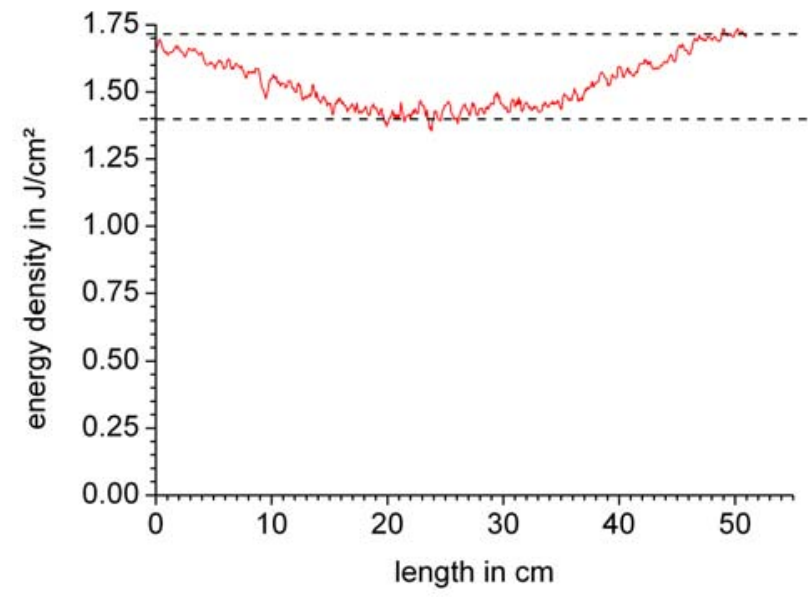

Fig. 17: profile of the energy density of a flash lamp array, determined along the yellow dotted line in figure 16

\section{Discussion}

The stability of the temperature after the excitation is crucial and will be considered in detail. The energy is injected into the plate from one side. During the energy flow from the source, the surface temperature will be overheated in comparison to the back side of the plate. When the source is removed, a temperature equalization takes place until the whole mass of the plate remains at the same temperature. This is the appropriate time to estimate the temperature difference for a calorimetric measurement. However, during the entire period of temperature equalization energy losses occur and distort the measurement result. Therefore, the equalization time should be as short as possible. This can be achieved by using very thin plates with small masses. On the other side, energy losses are roughly proportional to the temperature difference compared to the environment (if the difference is not higher than $10 \mathrm{~K}$ ). This effect arguments against very small masses. It can be concluded, that for a certain energy range, an optimized plate thickness should exist. The experimental results (figure 13 and figure 17) constrain that our sensors have a suited thickness for the relevant energy range. The fast decay immediately after the disappearing of the energy flow can be associated with the thermal equalization inside the sensor plate. The following period is characterized by a very slow decrease which is associated with thermal equalization processes between the sensor and the environment. Therefore, the beginning of this equalization period is the appropriate time to read out the temperature.

The measured linearity of the temperature rise in relation to the energy input (see figure 10 and 11) was surprisingly good and is in fact a proof for the robustness of the calorimetric sensor principle. However, one facet is still unclear. Referred to the geometrical data, the thermal capacity of both sensors should differ by a factor of almost 2.0 . During our experiments the observed temperature ratios were between 1.6 and 1.7 (see figure 9 and 13). This phenomenon became evident during the measurement series (figure 10 and 11) where the slopes of the fits should be a measure for the thermals capacities and the ratio was again significant smaller than factor 2.0. Some effects could be the reason:

- Higher energy losses at the thinner plate due to higher temperatures (see discussion above)

- Higher energy losses at the back side caused by better conducting glue connection to the wood stick

- Smaller emissivity and higher reflectivity of the black coating at the thinner plate

Regarding these points it is worthy to compare the measured temperature rises with theoretical estimated values.

The power sensor P 20 allows the determination of the true power density at a given point in space. Shifting the sensor zone along the optical axis of the blackbody radiator the predicted relation between distance and power density (Eq. 1) was found in principle (see figure 12). The calculated curve starts at the theoretical value of $94 \mathrm{~mW} / \mathrm{cm}^{2}$. The agreement is good for distances longer than $10 \mathrm{~cm}$, but at shorter distances a systematic deviation occurs. This can be explained by a simple geometrical consideration: The sensitive zone has an oval shape of $4.5 \mathrm{~cm}$ length and $2.5 \mathrm{~cm}$ height. So, even if the centre of the zone is exactly situated at the optical axis, outer parts of the zone do not receive the maximum power. This effect becomes stronger, if the sensitive zone is moved towards the radiating surface. Therefore, the power meter determines too low power densities at shorter distances systematically. However, based on the values obtained at longer distances, the 
power density can be interpolated into the region of shorter distances. For the case of our measurement series, conducted at $4.5 \mathrm{~cm}$ distance, a power density of $69 \mathrm{~mW} / \mathrm{cm}^{2}$ instead of $94 \mathrm{~mW} / \mathrm{cm}^{2}$ was estimated.

Fig. 18: comparison of calculated temperature rises and the experimental data, the variation of the energy input was realized by different blackbody temperatures in $4.5 \mathrm{~cm}$ distance, radiation time kept constant at $10 \mathrm{~s}$

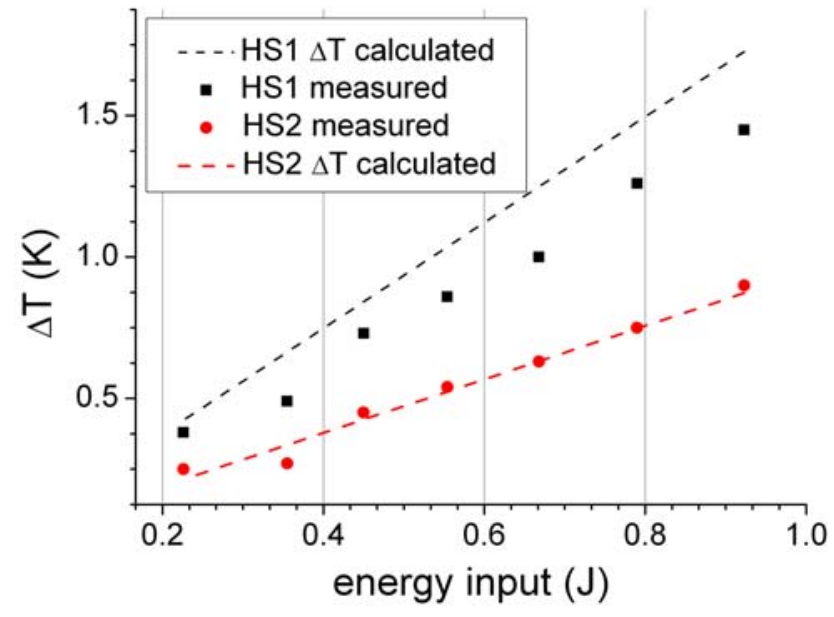

Using this geometrical correction factor the theoretical temperature rise was calculated for both sensors at different blackbody temperatures. Figure 18 includes the theoretical values and, again, the experimental data for comparison. The calculated curve for the HS 2 is in good agreement with the experimental data. In fact, this agreement is surprisingly, because the calculation of sensor signals did not consider any energy losses at all. One possible explanation could be an additional energy input caused by the white foam block (see figure 1). Figure 11 gives evidence for an unintentionally heating of the foam block holding the sticks of the sensors. Probably, the foam was much warmer during the radiation period. Therefore, it generated a secondary energy input at the back side of the sensor plates. This led to a compensation of energy losses at the front side. At this time it must be concluded, that we can only give a rough estimation of the measurement accuracy. Further investigations regarding the particular background of the sensor plates are needed. However, all obtained measurement data led to energy values in the excepted range, so the sensors should be suited for firstorder approximations.

The last part of the discussion deals with the actual energy yield of a usual flash lamp. The measurement result is shown in figure 14. After integrating the radial energy distribution over the circular plane a value of $1.1 \mathrm{~kJ}$ was obtained. This number seems to be small in relation the electric input energy of $6 \mathrm{~kJ}$. But, the following effects reduce the optical output that can be earned from a flash lamp:

- Conversion loss from electrical to optical power is $50 \%$ in the case of Xenon flash lamps [6]

- Arc discharge radiates symmetrically in all directions, the back reflector should collect as much as possible in forward direction, but the true collecting efficiency is not known, in reality it will be $50 \%$ roughly estimated

A third issue regards the measurement itself. The observed temperature profile was truncated at the edge of the used paperboard corresponding to a distance of $50 \mathrm{~cm}$ to the centre of the circular shaped energy distribution. But, the energy density value of $50 \mathrm{~mJ} / \mathrm{cm}^{2}$ at the cut off indicates that a part of radiated energy outside of the $50 \mathrm{~cm}$ radius. By contrast, considering the geometrical shape of the reflector, the diameter of the excited circular zone should be $90 \mathrm{~cm}$. In fact, the radiated zone is larger and the performed energy estimation did not include the entire zone. In view of these circumstances the estimated value of $1.1 \mathrm{~kJ}$ is quite reasonable, and so are the estimated values for energy densities.

\section{Conclusions}

The objective of the investigations was establishing a suited procedure in the field of active thermography to estimate the energy distribution generated by flash lamps. The proposed method bases on the combination of a large area intensity detector with a point shaped absolute energy meter. The experimental problem is the correct estimation of the energy irradiated by a source with a diverging radiation. In order to solve this problem, a simple calorimetric sensor without any kind of housing was presented. The suitability of the calorimetric principle could be demonstrated in a series of measurements. Then, the applicability of the whole procedure was proven for the case of a single flash lamp with $6 \mathrm{~kJ}$ energy consumption. The detected energy, measured in a distance of $35 \mathrm{~cm}$, was $1.1 \mathrm{~kJ}$ inside a circular shaped zone with $50 \mathrm{~cm}$ radius. The corresponding energy density was in good agreement with the result published by another group [4]. So, it can be concluded, that the proposed procedure works quite well and has the potential to become a valuable tool in the field of flash thermography.

In order to establish the method for more precise energy determination, a lot of detailed investigations are necessary. This concerns the entire sensor design as well as the influence of the particular background. Further, the suitability of the procedure for the characterization of other extended diverging radiating sources like halogen lamps, lamp arrays and LED arrays has to be shown. 


\section{References}

[1] Avdelidis, N.P., Ibarra-Castanedo, C., Maldague, X., Marioli-Riga, Z.P.,Almond, D.P.: A thermographic comparison study for the assessment of composite patches, Infrared Physics \& Technology 45 (2004), pp. 291-299

[2] Cernuschi, F., Lamperti, M., Marchesi, R., Russo, A.: Characterization of spatial light distribution of flash lamp systems. In: Proceddings og QIRT 1996, Eurotherm Series 50, Editioni ETS, Pisa 1997, pp. 15-19

[3] Richter, R., Maierhofer, C., Kreutzbruck, M. and Schilling, M.: Numerical method of active thermography for the reconstruction of back wall geometry, 6th Workshop NDT in Progress 2011, pp. 245-255

[4] Pickering, S. \& Almond, D.: Matched excitation energy comparison of the pulse and lock-in thermography NDE techniques, NDT \& E International, 2008, 41, 501 - 509

[5] Savage, M. \& Heilman, J.: Infrared calibration of net radiometers and infrared thermometers, Agricultural and Forest Meteorology, 2009, 149, 1279 - 1293

[6] Product brochure of PerkinElmer optoelectronics: High Performance Flash and Arc Lamps, http://www.htds.fr/doc/optronique/sourcesLumineuses/LampesFlash.pdf, p. 10 